\title{
Review
}

\section{Validity of Intermittent Infusion Hemodiafiltration}

\author{
Michio Mineshima $^{\text {a }}$ Kei Eguchi ${ }^{b}$ \\ ${ }^{a}$ Department of Clinical Engineering, Tokyo Women's Medical University, Tokyo, Japan; \\ ${ }^{b}$ Department of Clinical Engineering, Tohoku Medical and Pharmaceutical University, Miyagi, Japan
}

\section{Keywords}

Intermittent infusion hemodiafiltration · Backfiltration ·

Plasma refilling $\cdot$ Peripheral circulation

\begin{abstract}
Background: Intermittent infusion hemodiafiltration (IHDF) has been developed to prevent a rapid drop in blood pressure during a dialysis session and to improve peripheral circulation. In Japan, >10,000 dialysis patients underwent treatment with I-HDF in 2017, and the number of dialysis patients is increasing year by year. I-HDF involves the intermittent infusion of ultrapure dialysis fluid or sterile nonpyrogenic substitution fluid, for example, at a volume of $200 \mathrm{~mL}$ and a rate of $150 \mathrm{~mL} / \mathrm{min}$ by backfiltration every $30 \mathrm{~min}$ during treatment. The total infusion volume can therefore be estimated at $200(\mathrm{~mL}) \times 7$ (infusions) or $1.4 \mathrm{~L} /$ session. I-HDF may be regarded as online HDF with a very small replacement volume. Summary: Several clinical trials of I-HDF have been conducted in Japan. (1) In a 2007 study, despite there being no differences noted in the volume of water removal between hemodialysis (HD) and I-HDF, a significantly lower rate of reduction in the time-averaged blood volume was seen in I-HDF than in HD, so the plasma refilling rate was greater during I-HDF. (2) In a 2015 study, at 13 weeks after a switch from HD, I-HDF was found to be significantly superior to HD in terms of the incidence of events needing interven-
\end{abstract}

tion by medical staff. However, significantly lower blood $\beta_{2^{-}}$ microglobulin (MG) and $a_{1}-M G$ levels were observed in the predilution online HDF (pre-HDF) group than in the I-HDF group, and the amount of albumin leak was lower in the IHDF group than in the pre-HDF group. (3) In a 2017 study, compared with HD, I-HDF was associated with a reduced number of interventions for intradialytic hypotension and less severe tachycardia, suggesting less sympathetic stimulation during I-HDF. Key messages: I-HDF is a valid treatment option because it is associated with an increased plasma refilling rate and fewer interventions needed by medical staff.

(c) 2019 S. Karger AG, Basel

\section{Introduction}

In 2007, we reported our development of intermittent infusion hemodiafiltration (I-HDF) as a procedure to prevent a rapid decrease in blood pressure (BP) during a dialysis session and to improve peripheral circulation. In I-HDF, ultrapure dialysis fluid or sterile nonpyrogenic substitution fluid is infused intermittently to increase the patient's circulating blood volume (BV) during dialysis [1-3]. The aims of intermittent infusion are two-fold: to reduce the risk of a decrease in $\mathrm{BP}$ during treatment by preventing a rapid drop in $\mathrm{BV}$ in response to removal of excess water and to increase transport of water and sol-

\section{KARGER}

(c) 2019 S. Karger AG, Basel

E-Mail karger@karger.com

www.karger.com/bpu
Michio Mineshima

Department of Clinical Engineering

Tokyo Women's Medical University

8-1 Kawada-cho, Shinjuku-ku, Tokyo 162-8666 (Japan)

E-Mail mmine@ twmu.ac.jp 
Fig. 1. Time course of change in blood volume ratio $(\triangle \mathrm{BV})$ estimated from the hematocrit monitor and the peripheral blood flow rate $\left(\mathrm{PQ}_{\mathrm{B}}\right)$ from the laser flow meter [3].

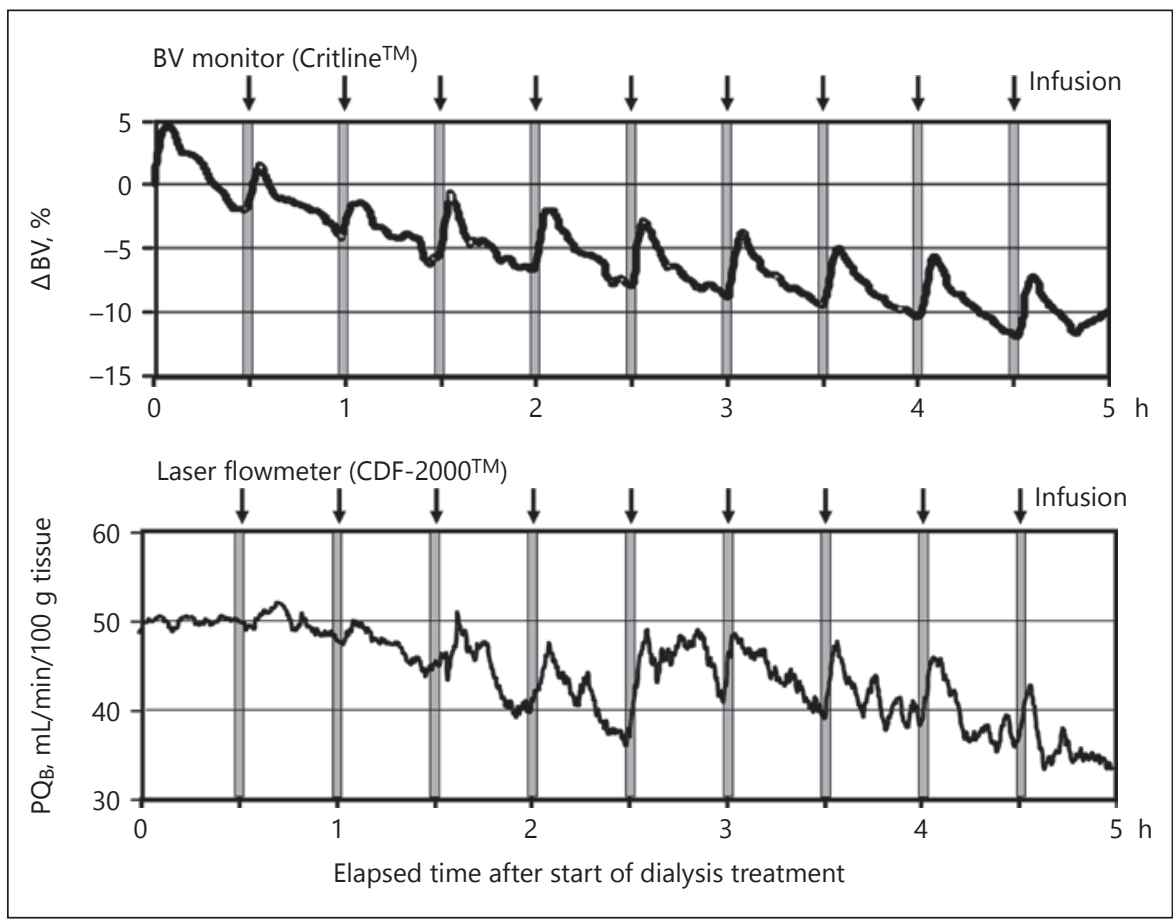

utes from the extravascular space to the intravascular space (i.e., plasma refilling) by improving the patient's peripheral circulation.

There are several types of I-HDF: (1) I-HDF using saline solution, (2) I-HDF using sterile substitution fluid, (3) I-HDF using ultrapure dialysis fluid by rapid infusion mode of a dialysis machine, and (4) I-HDF utilizing backfiltration of ultrapure dialysis fluid through a dialysis membrane. Notably, in type (4) I-HDF, backflushing of the membrane with purified dialysate is expected to reduce the fouling effect caused by attachment of proteins to the membrane as a result of ultrafiltration [4]. In Japan, >10,000 dialysis patients underwent treatment with type (4) I-HDF in 2017, with the number of dialysis patients undergoing such treatment is growing year by year.

Typically, type (4) I-HDF involves the infusion of ultrapure dialysis fluid at a volume of $200 \mathrm{~mL}$ and a rate of $150 \mathrm{~mL} / \mathrm{min}$ by backfiltration every $30 \mathrm{~min}$ during treatment. Alternately, the same volume of body fluid is filtered after each infusion in addition to removal of excess water. The first infusion is performed $30 \mathrm{~min}$ after the start of treatment, and the last is performed $30 \mathrm{~min}$ before the end of treatment. Therefore, the total infusion volume could be estimated at $200(\mathrm{~mL}) \times 7$ (infusions), or $1.4 \mathrm{~L} /$ session. I-HDF may therefore be regarded as online HDF with a very small replacement volume.
Figure 1 shows the time course of the change in $\mathrm{BV}$ ratio $(\triangle \mathrm{BV})$ estimated from the hematocrit monitor and the peripheral blood flow rate $\left(\mathrm{PQ}_{\mathrm{B}}\right)$ from the laser flow meter. Increases in $\triangle \mathrm{BV}$ and $\mathrm{PQ}_{\mathrm{B}}$ were observed after each infusion in many patients [3].

\section{Clinical Studies}

To date, several clinical trials of I-HDF have been conducted in Japan.

(1) A prospective, multicenter, single-treatment, crossover trial that compared I-HDF with backfiltration of purified dialysis fluid and typical hemodialysis (HD) was reported in 2007 [1]. Figure 2 shows a comparison between HD and I-HDF for the total volume of water removal $\left(\mathrm{V}_{\mathrm{WR}}\right)$ and time-averaged $\mathrm{BV}$ reduction rate $(\mathrm{BV})$ [3]. Despite there being no difference in $V_{W R}$ value between the HD and I-HDF treatments, a significantly lower rate of reduction of the time-averaged BV was shown in I-HDF than in HD, so the plasma refilling rate was greater during I-HDF. Furthermore, the clear space (CS) values for inorganic phosphate and $\alpha_{1}$-microglobulin (MG) were significantly higher during I-HDF than during HD.

(2) A prospective, multicenter, parallel-group comparative trial of I-HDF and online predilution HDF (pre- 
Fig. 2. Comparison between standard hemodialysis and I-HDF for total volume of water removal $\left(\mathrm{V}_{\mathrm{WR}}\right)$, and time-averaged blood volume reduction rate (RR[BV]) [3]. I-HDF, intermittent infusion hemodiafiltration; HD, hemodialysis.

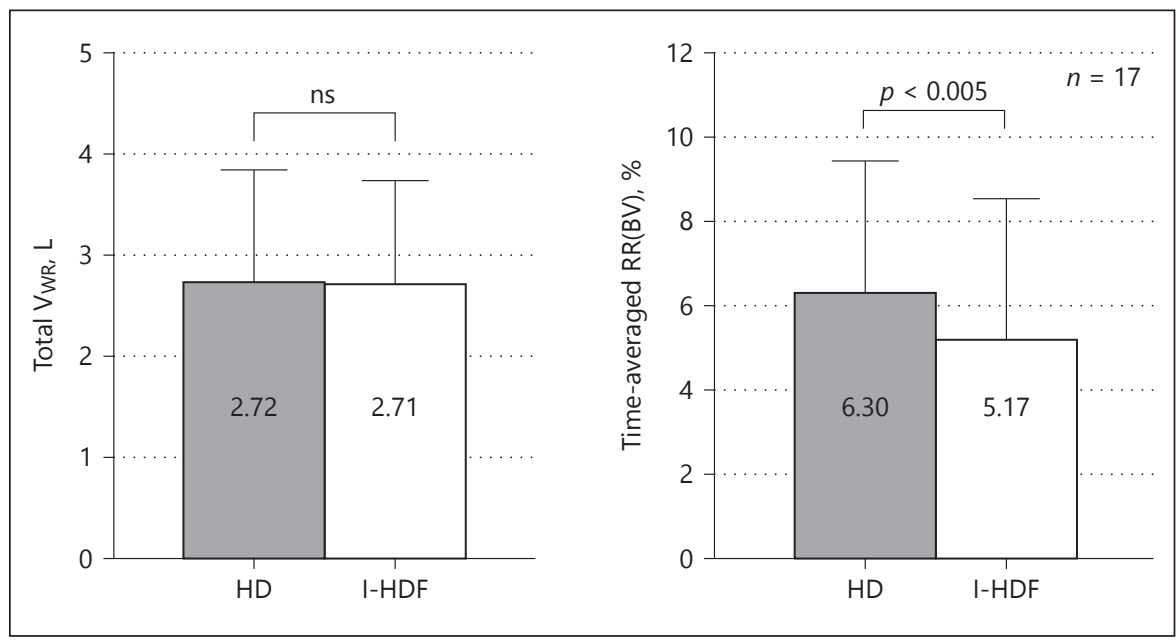

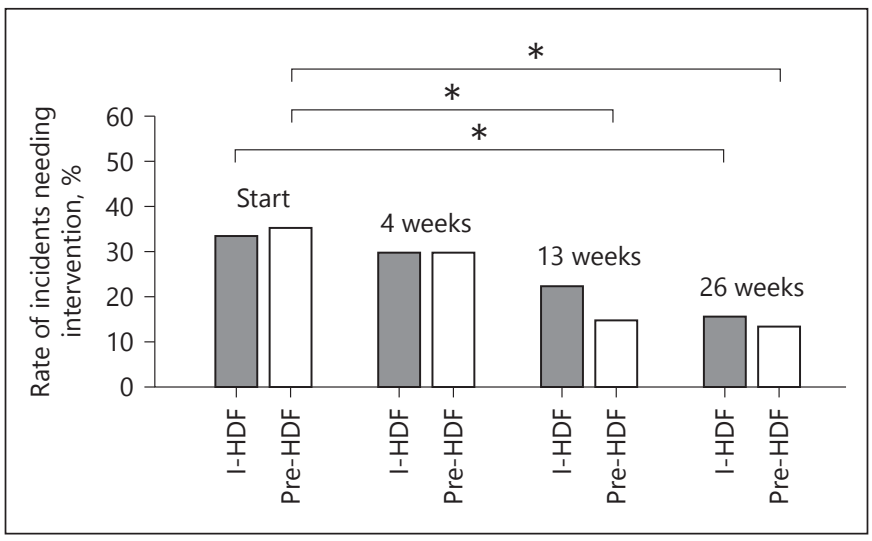

Fig. 3. Rate of incidents needing intervention per week at the start of treatment and at 4,13 , and 26 weeks later $[5,6]$. I-HDF vs. preHDF (overall): not significant (Mantel extension test). Start of treatment vs. 13 weeks for pre-HDF, Start vs. 26 weeks for I-HDF and pre-HDF: ${ }^{*} p<0.05$ ( $\chi^{2}$ test). I-HDF, intermittent infusion hemodiafiltration; pre-HDF, predilution HDF.

HDF) was reported in 2015 [5, 6]. Online predilution $\mathrm{HDF}$ is a more popular modality in Japan than in Europe, and this is because of the relatively lower blood flow rate. The patients were divided into 2 groups matched for age, dry weight, and prevalence of diabetes. A total of $36 \mathrm{pa}-$ tients (18 pairs) provided informed consent and were enrolled in the study. Changes in the clinical parameters, quality of life, and solute removal characteristics were evaluated at 4,13 , and 26 weeks after switching from conventional HD to either of the trial therapies.

Figure 3 shows the rate of incidents requiring intervention by medical staff per week at the start of treatment and at 4,13 , and 26 weeks later $[5,6]$. There was no sig- nificant difference between the 2 groups in the amount of change in mean systolic BP or in the incidence of events needing intervention in the first week after switching from HD. However, BP became more stable as time passed, and the incidence of events requiring intervention decreased in both study groups when compared with the incidence recorded for conventional HD. At 13 weeks after the switch, I-HDF was significantly superior to HD in terms of the incidence of events needing intervention. In other words, at least 3 months were needed to establish the superiority of I-HDF over HD.

However, the results of blood tests performed at baseline and at 4,13, and 26 weeks after the start of the study revealed significantly lower blood $\beta_{2}-\mathrm{MG}$ and $\alpha_{1}-\mathrm{MG}$ levels in the pre-HDF group than in the I-HDF group. Furthermore, the clear space value for $\beta_{2}-\mathrm{MG}$ and amount of albumin leak were lower in the I-HDF group than in the pre-HDF group. The latter finding could reflect the difference in replacement volume between I-HDF $(1.4 \mathrm{~L})$ and pre-HDF $(44.9 \pm 4.0 \mathrm{~L})$. As shown in Figure 4 , in terms of the amount of albumin leak, I-HDF had significantly lower albumin loss compared with preHDF.

(3) A prospective, multicenter, crossover trial that compared conventional HD and I-HDF with backfiltration of purified dialysis fluid was reported in 2017 [7]. This study involved 68 patients who were prone to a drop in BP while alternating treatment between I-HDF and HD for 4 weeks each. The rate of interventions was found to be lower for intradialytic hypotension associated with increased intradialytic BP and less severe tachycardia, suggesting less sympathetic stimulation during I-HDF. 


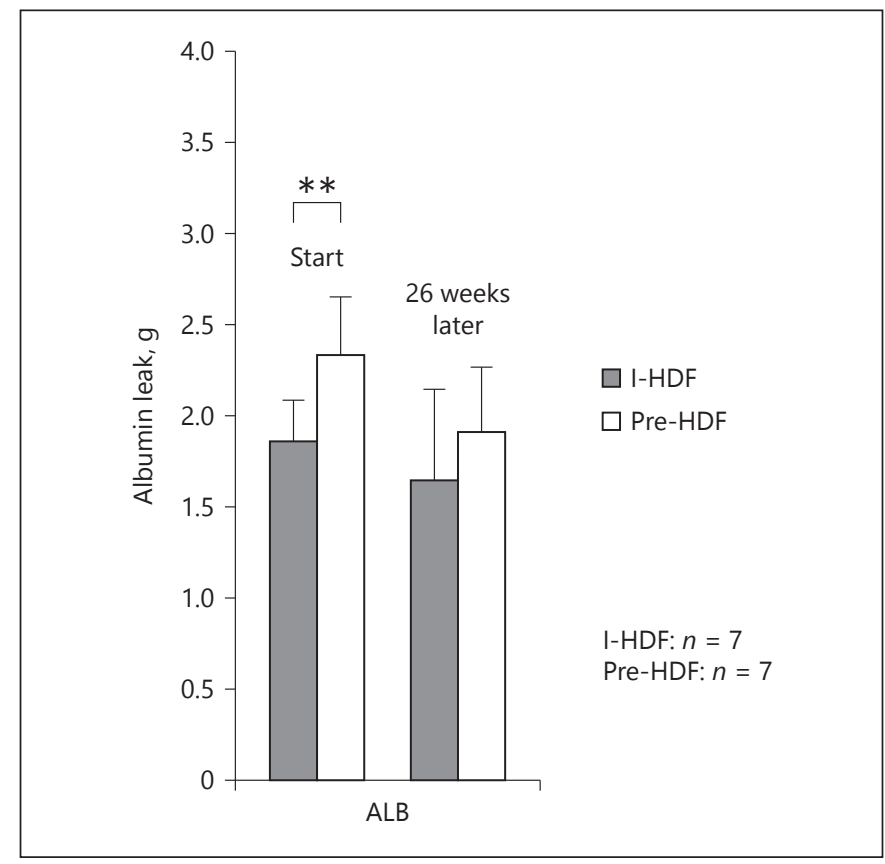

Fig. 4. Albumin leak at the start of treatment and at 26 weeks later $[5,6] .{ }^{* *} p<0.01$. I-HDF, intermittent infusion hemodiafiltration; pre-HDF, predilution HDF.

Although the abovementioned studies have, in part, established the clinical effectiveness of I-HDF, the indications for I-HDF and the adequate infusion pattern for each patient have not been elucidated. Further clinical studies will be required.

\section{Conclusions}

I-HDF is a valid treatment option because it is associated with an increased plasma refilling rate and fewer interventions needed by medical staff.

\section{Acknowledgment}

None.

\section{Statement of Ethics}

No ethics approval was required.

\section{Disclosure Statement}

The authors have no conflicts of interest to declare.

\section{Funding Sources}

This research received no specific grant from any funding agency in the public, commercial, or not-for-profit sectors.

\section{Author Contributions}

M.M. prepared the manuscript as a principal author. K.E. discussed and coauthored the manuscript.

\section{References}

1 Eguchi K, Ikebe M, Konno Y, Yamada Y, Kaneko I, Mineshima M, et al. Development and clinical effectiveness of intermittent infusion hemodiafiltration (I-HDF) as a new therapy. J Jpn Soc Dial Ther. 2007;40(9):769-74.

2 Eguchi K, Miyao M, Yamada Y, Konno Y, Kaneko I, Mineshima M, et al. Clinical assessment of intermittent infusion dialysis (I-HD) using backfiltration of ultrapure dialysis fluid by an automated machine. J Jpn Soc Dial Ther. 2009;42(9):695-703.

3 Mineshima M, Eguchi K. Development of intermittent infusion hemodiafiltration using ultrapure dialysis fluid with an automated dialysis machine. Blood Purif. 2013;35(suppl 1): $55-8$.
4 Miyazaki M, Fukuda M, Uezumi S, Yoshida $\mathrm{H}$, Takahashi T, Watanabe T, et al. Effect of operating conditions on solute removal amount during a hemodialysis using several hemodialyzers with enhanced internal filtration and automated dialysis console produced by JMS. Kidney and Dialysis. High Performance Membrane. 2006;61:126-32.

5 Mineshima M, Eguchi K, Shishido K, Takahashi S, Kubo T, Kawaguchi H, et al. Clinical effectiveness of intermittent infusion hemodiafiltration using backfiltration of ultrapure dialysis fluid compared with predilution online hemodiafiltration; a prospective, multicenter and controlled trial. J Jpn Soc Dial Ther. 2015;48(6):351-60.
6 Mineshima M, Eguchi K, Shishido S, Takahashi S, Kubo T, Kawaguchi H, et al. Clinical effectiveness of Intermittent Infusion Hemodiafiltration using backfiltration of ultrapure dialysis fluid compared with predilution online hemodiafiltration. In: Kawanishi $\mathrm{H}$, Takemoto Y, editors. Scientific aspects of dialysis therapy, JSDT/JSFA anniversary edition. Contrib Nephrol. Volume 189. Basel: Karger; 2017. pp. 24-9.

7 Koda Y, AoikeI, Hasegawa S, Osawa Y, Nakagawa Y, Iwabuchi F, et al. Feasibility of intermittent back-filtrate infusion hemodiafiltration to reduce intradialytic hypotension in patients with cardiovascular instability: a pilot study. Clin Exp Nephrol. 2017 Apr;21(2):324-32. 